Cell Research (2003); 13(5):351-359

http://www.cell-research.com

\title{
THE IN VITRO RECONSTITUTION OF NUCLEOSOME AND ITS BINDING PATTERNS WITH HMG1/2 AND HMG14/17 PROTEINS
}

Shu Bing Zhang ${ }^{1 *}$, Jian Huang1* ${ }^{*}$, Hui Zhao ${ }^{1 *}$, Yi Zhang ${ }^{2}$, Chun Hui Hou $^{1}$, Xiao Dong Cheng ${ }^{1}$, Chu Jiang ${ }^{1}$, Min Qian $\mathrm{Li}^{2}, \mathrm{Jun} \mathrm{Hu}{ }^{2}$, Ruo Lan QIAN ${ }^{1 * *}$

1 Group of Globin Gene Expression and Regulation, State Key Laboratory of Molecular Biology, Institute of Biochemistry and Cell Biology, Shanghai Institutes for Biological Sciences, Chinese Academy of Sciences, Shanghai 200031,China. Email:qianlab@sunm.shcnc.ac.cn

2 Shanghai Institute of Nuclear Research, Chinese Academy of Sciences, Shanghai 201800, China

\begin{abstract}
Using atomic force microscopy (AFM), the dynamic process of the in vitro nucleosome reconstitution followed by slow dilution from high salt to low salt was visualized. Data showed that the histone octamers were dissociated from DNA at $1 \mathrm{M} \mathrm{NaCl}$. When the salt concentration was slowly reduced to $650 \mathrm{mM}$ and $300 \mathrm{mM}$, the core histones bound to the naked DNA gradually. Once the salt concentration was reduced to $50 \mathrm{mM}$ the classic "beads-on-astring" structure was clearly visualized. Furthermore, using the technique of the in vitro reconstitution of nucleosome, the mono- and di- nucleosomes were assembled in vitro with both HS2core (-10681 to -10970 bp) and NCR2 (-372 to -194 bp) DNA sequences in the 5'flanking sequence of human b-globin gene. Data revealed that HMG $1 / 2$ and HMG14/17 proteins binding to both DNA sequences are changeable following the assembly and disassembly of nucleosomes. We suggest that the changeable binding patterns of HMG 14/17 and HMG1/2 proteins with these regulatory elements may be critical in the process of nucleosome assembly, recruitment of chromatin-modifying activities, and the regulation of human b-globin gene expression.
\end{abstract}

Keywords: atomic force microscopy, HMG proteins (HMG1/2 and HMG14/17), human b-globin gene, in vitro reconstitution, nucleosome.

\section{INTRODUCTION}

In eukaryotic cells, gene expression is not determined solely by DNA itself. It is influenced by proteins that package DNA into chromatin. However, the molecular mechanisms by which chromatin plays its dynamic role in regulating gene activity are poorly understood. Much is now known concerning the structure of the nucleosome, the fundamental repeating unit of eukaryotic chromatin. The physical properties of nucleosome depend on solution conditions such as ionic strength and divalent-ion concentration as well as on histone-modifi-

* Authors contributed equally to the work.

**Correspondence to Prof. Ruo Lan QIAN E-mail: qianlab@sunm. shcnc.ac.cn Tel: 86-21-54921354 Fax: 86-21-54921011

Abbreviations: HMG, High Mobility Group proteins; AFM, Atomic Force Microscopy; HS2 core, the core DNA sequence of DNaseI HyperSensitive site 2; NCR2, Negative Control Region 2; EMSA,Electrophoresis Mobility Shift Assay.

Received Sept-28-2002 Revised June-20-2003 Accepted-Aug-25-2003 cation state. Recent evidences show that acetylation or methylation of histone proteins can alter the patterns of gene activity. In living cells the process of nucleosome assembly and disassembly, recruitment of chromatinmodifying activities, and regulation of gene activities is very complicated and dynamic.

In our previous studies the chromatin folding patterns in chicken erythrocytes were revealed by AFM. At the first level of DNA packing, our data showed that an extended beads-on -a-string structure (width of $\sim 15-20 \mathrm{~nm}$, height of $\sim 2-3 \mathrm{~nm}$ for each individual nucleosome) could be consistently observed. Furthermore, superbeads (width of $\sim 40 \mathrm{~nm}$, height of $\sim 7 \mathrm{~nm}$ ) arranged with irregular distance along the extended beads -on-a-string were demonstrated[1]. In addition, the structure of the nucleosome core particle of chromatin in chicken erythrocytes was further revealed by using AFM. The $\sim 146$ bp of DNA wrapped twice around the core histone octamer 
were clearly visualized. Both the ends of entry/exit of linker DNA were also demonstrated[2].

In an attempt to gain further insight into the dynamic processes of the in vitro nucleosome reconstitution, the linker histones-depleted oligo-nucleosomes were prepared from chicken erythrocytes. The process of the in vitro nucleosome reconstitution followed by slow dilution from high salt to low salt was imaged with AFM. Once the salt concentration had been reduced to $50 \mathrm{mM}$, the classic "beads -on-a-string" structure was clearly visualized (width of $\sim 18 \mathrm{~nm}$, height of $\sim 2-4 \mathrm{~nm}$ for each individual nucleosome).

Furthermore, using the technique of the in vitro reconstitution of nucleosome, the mono-and di-nucleosomes were assembled with both the core DNA sequence of DNase I hypersensitive site II (HS2core DNA sequence, -10681 to $-10970 \mathrm{bp}, \sim 290 \mathrm{bp}$ ) in LCR and NCR2 (-372 to $-194 \mathrm{bp}, \sim 178 \mathrm{bp})$ DNA sequence in 5 ' flanking sequence of human b-globin gene. HS2 is an important control element within b-globin LCR. It functions as a typical enhancer. While, NCR2 is a silencer in the $5^{\prime}$ flanking sequence of human b-globin gene[3]. It is our further aim to examine whether HMG proteins (HMG1/ 2 and HMG14/17) can bind to both the HS2 core and the NCR2 DNA fragments presented in different states (either in the naked DNA or in the in vitro reconstituted nucleosomal DNA). HMG proteins are abundant chromosomal non-histone proteins. It has been shown that HMG proteins may play an important role in the structure and function of chromatin. However, their role in the regulation of eukaryotic gene transcription remains to be established. We show here that HMG $1 / 2$ proteins are able to bind to both the naked HS2core and NCR2 DNA sequences. While, these proteins are unable to bind to both HS2core and NCR2 DNA sequences assembled in vitro into nucleosomes with the core histone octamers transferred from the linker histones-stripped oligonucleosomes. In contrast, HMG14/17 proteins are unable to bind to both naked HS2core and NCR2 DNA sequences, whereas they are capable of binding to the nucleosomes assembled in vitro with HS2 core and NCR2 DNA fragments. It seems that the binding patterns of HMG1/2 and HMG14/17 proteins to both the distal and proximal regulatory elements of human b-globin gene are changeable following the assembly and disassembly of nucleosomes. Results presented here might provide some important clues for both the process of in vitro reconstitution of nucleosomes and the biological functions of
HMG proteins (HMG1/2 and HMG14/17 proteins) in the structure of chromatin and in the regulation of globin gene expression.

\section{MATERIALS AND METHODS}

\section{Preparation of the linker histones -stripped oligo- nucleosomes}

Nuclei were prepared from chicken erythrocytes and processed as described by Lutter[4]. These nuclei were then digested with micrococcal nuclease ( 40 units $/ \mathrm{ml}$ ) in the presence of $1 \mathrm{mMCaCl} 2$ for $5 \mathrm{~min}$ at $37^{\circ} \mathrm{C}$. The reaction was stopped by adjustment to $2 \mathrm{mM}$ EDTA. The solution is centrifuged for $10 \min (3000 \mathrm{x} \mathrm{g})$ at $4{ }^{\circ} \mathrm{C}$. The pellet was resuspended and lysed by pipetting gently up and down in $2.5 \mathrm{ml}$ of lysis solution (10 $\mathrm{m} M$ Tris-HCl pH 7.5, 0.2 $\mathrm{m} M$ EDTA and $0.2 \mathrm{mM}$ PMSF). The solution was kept on ice for at least $30 \mathrm{~min}$ and was shaken gently to release the chromatin into solution. Then, the solution was centrifuged again for $10 \mathrm{~min}(3000 \mathrm{xg})$ at $4{ }^{\circ} \mathrm{C}$. The supernatant was adjusted to $0.65 \mathrm{M} \mathrm{NaCl}$ and then applied to a sepharose 4B column $(2 \mathrm{~cm} \times 80 \mathrm{~cm})$ that had been equilibrated with $0.65 \mathrm{M} \mathrm{NaCl}$, $5 \mathrm{~m} M$ Tris- $\mathrm{HCl}$ (pH7.5), 0.2 mM 2-mercaptoethnol. Fractions containing $\mathrm{A}_{260}$ absorbing material were collected. Small portions of representative column fractions were electrophoresed on $18 \%(\mathrm{w} / \mathrm{v})$ acrylamide and $0.1 \%(\mathrm{w} / \mathrm{v})$ SDS to examine the presence of histone $\mathrm{H} 1$ or H5.

\section{The in vitro reconstitution of nucleosome and AFM analysis}

The nucleosome reconstitution was performed according to the method of Drew et al. [5]. The solution containing the linker histones (H1 and $\mathrm{H} 5)$-stripped oligo-nucleosomes was adjusted to $1 \mathrm{M} \mathrm{NaCl}$ and $1 \mathrm{~m} M$ PMSF, followed by slow dilution from high salt $(1 M \mathrm{NaCl})$ to low salt $(50 \mathrm{~m} M \mathrm{NaCl})$, and tapping mode images were obtained on a nanoscope III AFM (Digital instruments, Santa Barbara, CA), using nanoprobe silicon tips, at a scan rate of $1.5 \mathrm{~Hz}$.

\section{The in vitro reconstitution of nucleosomes with both}

\section{the labeled HS2core and NCR2 DNA fragments}

The H1 and H5-depleted oligo-nucleosomes were prepared from the nuclei of chicken erythrocytes. The labeled HS2core and NCR2 DNA fragments were reconstituted into nucleosomes with the core histone octamers transferred from the $\mathrm{H} 1$ and H5-depleted oligonucleosomes[6]. Samples were electrophoresed on $5 \%$ acrylamide gel and autoradiographed.

\section{Gel mobility shift assay}

HMG14/17and HMG1/2 proteins were prepared from the nuclei of chicken erythrocytes according to the method of Goodwin et al [7]. The protein concentration was determined by Bradford's method [8]. Both HS2core DNA fragment (-10681 to $-10970 \mathrm{bp}$ ) and NCR2 DNA fragment (-372 to $-194 \mathrm{bp}$ ) in 5'flanking sequence of human b-globin gene were prepared with restriction enzymes and labeled with $\gamma-32 \mathrm{P}-$ 
ATP. Gel mobility shift assay was performed according to the method described previously[9]. The HMG proteins were incubated with the DNA probe in $25 \mu \mathrm{L}$ reaction mixture $(25 \mathrm{mM}$ Tris- $\mathrm{HCl}, \mathrm{pH} 7.9,0$. $05 \mathrm{~m} M$ EDTA, $0.5 \mathrm{~m} M$ DTT, $0.5 \mathrm{~m} M$ PMSF and $5 \%$ glycerol) on ice for $45 \mathrm{~min}$ with addition of enough polydI-polydC as non-specific competitor. The binding of HMG proteins to the DNA probe was resolved by $6 \%$ non-denaturing polyacrylamide gel.

\section{RESULTS}

\section{Identification of the linker histones-stripped} oligonucleosomes isolated from chicken erythrocytes

To study in vitro nucleosome reconstitution, the linker histones-stripped oligonucleosomes were prepared from chicken erythrocytes. Small portions of representative column fractions were electrophoresed on $18 \%(\mathrm{w} / \mathrm{v})$ acrylamide and $0.1 \%(\mathrm{w} / \mathrm{v}) \mathrm{SDS}$ to examine whether the linker-histones (H1 or H5) were stripped. Data revealed that linker histones ( $\mathrm{H} 1$ or $\mathrm{H} 5$ ) could not be detected in samples examined (Fig 1, lanes 1-2 and 4-6). Then, the linker histones-depleted oligo-nuclosomes (containing $\sim 15$ nucleosomes) were used for in vitro reconstituton tests.

Visualization of the dynamic process of in vitro nucleosome reconstitution by AFM

In order to reveal the process of in vitro nucleosome reconstitution, the samples of in vitro nucleosome reconstitution at different concentrations of $\mathrm{NaCl}$ were prepared and imaged by using AFM. Results demonstrated that the histone octamers were dissociated from DNA

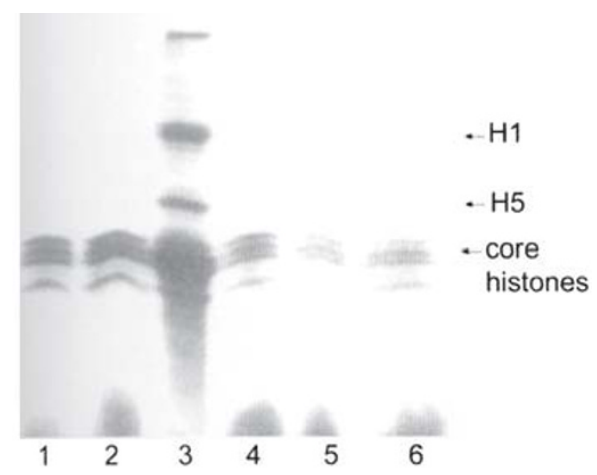

Fig 1. Identification of histone proteins in the linker histonesstripped oligo-nucleosomes. Small portions of representative column fractions were electrophoresed on SDS-PAGE. Lanes 1-2 and 4-6, The linker-histones (H1 and H5) could not be detected in the samples examined; lane 3, total histones isolated from chicken erythrocytes as a marker. at $1 \mathrm{M} \mathrm{NaCl}$ (Fig 2A). Furthermore, when the concentration of $\mathrm{NaCl}$ was slowly reduced to $650 \mathrm{mM}$ and 300 $\mathrm{mM}$, the histones were capable of binding to the naked DNA again (Fig 2B and Fig 2C). Then, the oligo-nucleosome fibers were folded into the compact fibers when the concentration of $\mathrm{NaCl}$ was reduced to $100 \mathrm{mM}$ (Fig 2D). Furthermore, these fibers were further loosed when the salt concentration was diluted to $50 \mathrm{mM}$ (Fig 2E). The classic " beads -on-a-string" structure was clearly visualized (height of $\sim 2-4 \mathrm{~nm}$, width of $\sim 18 \mathrm{~nm}$ for each individual nucleosome). Results demonstrated that the in vitro reconstituted nucleosomes did not differ in morphology from native ones[1].

\section{Identification of in vitro reconstitution of nucleo-} somes with the HS2core DNA fragments

The Locus Control Region (LCR) is located at $6-22 \mathrm{~kb}$ upstream of human b-globin gene. It is characterized by four DNase I hypersensitive sites (HS1, HS2, HS3 and HS4). HS2 is a typical enhancer. The unlabeled HS2core DNA fragment (-10681 to -10970 bp, 290 bp) was used to reconstitute in vitro into nucleosomes with the core histone octamers transferred from the $\mathrm{H} 1$ and $\mathrm{H} 5$-depleted oligo-nucleosomes. The in vitro reconstituted mono- and di- nucleosomes could be visualized by using AFM (Fig 3A and Fig 3B). The thin arrows point to the reconstituted mono-nucleosomes and the thick arrows indicate the reconstituted di-nucleosomes. The length of the used HS2core DNA fragment was only $290 \mathrm{bp}$. Therefore the linker DNA between the reconstituted di-nucleosomes was very short, while the DNA sequence flanking the reconstituted mono-nucleosomes was quite long.

Furthermore, the mono- and di -nucleosomes were assembled in vitro with the $\gamma-32 \mathrm{P}-\mathrm{ATP}$ labeled HS2core DNA fragment (Fig 4, lanes 2-4, bands A and B). The band of the reconstituted di-nucleosomes (Fig 4, lanes 24 , band A) was very similar to that of the native di-nucleosome from chicken erythrocytes (Fig 4, lane 5, band A). However, the band of the in vitro reconstituted mononucleosomes (Fig 4, lanes 2-4, band B) migrated significantly slower than that of the native mono-nucleosome from chicken erythrocytes (Fig 4, lane 5, band C). The reason is that the length of the labeled HS2core DNA fragment ( $290 \mathrm{bp}$ ) is longer than that of the native mononucleosomal DNA $(\sim 146 \mathrm{bp})$ isolated from chicken erythrocytes. Data further confirmed that the in vitro reconstitution of nucleosome was successful. 

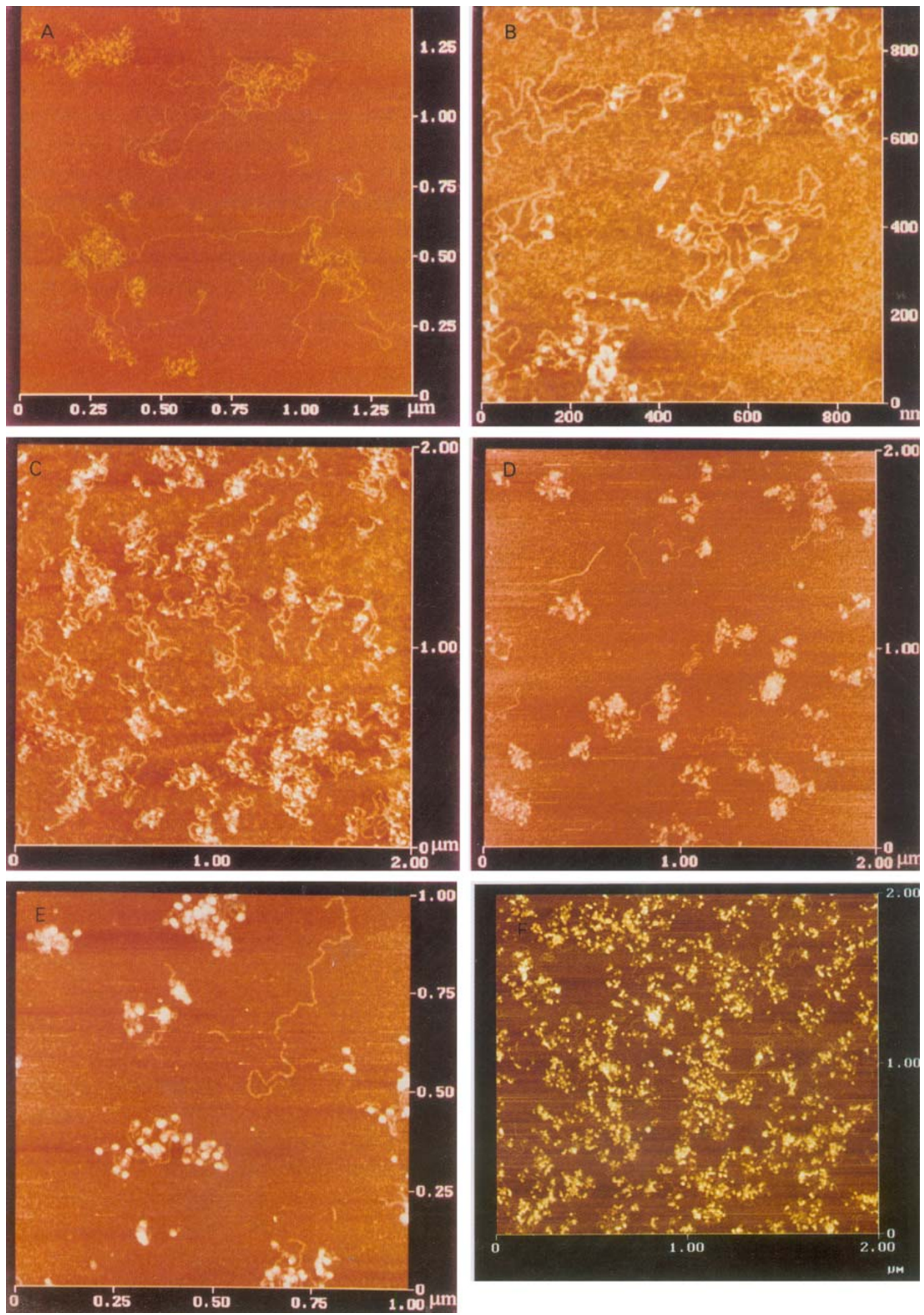

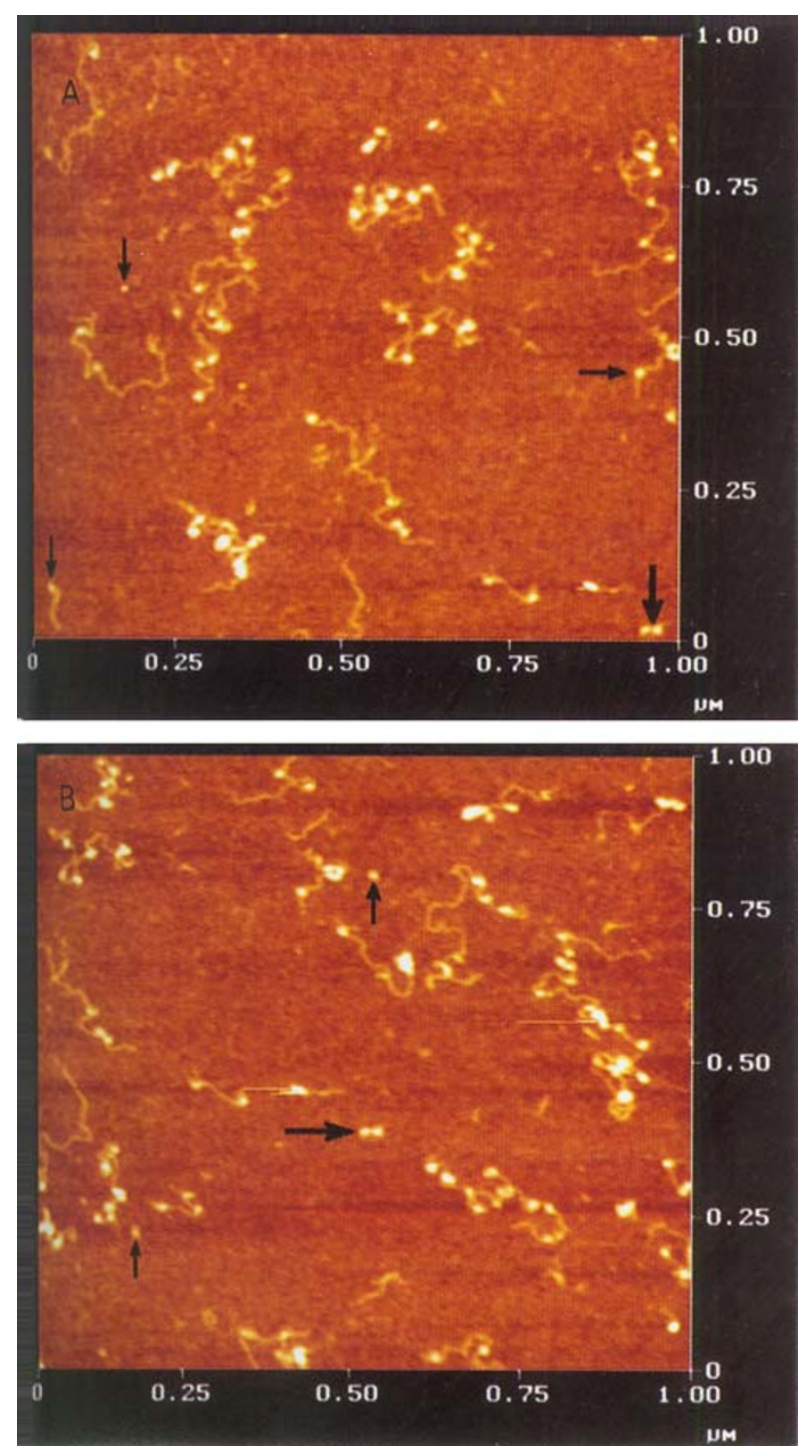

Fig 3. (A and B). AFM images of the in vitro reconstitution of nucleosomes with the unlabeled HS2core DNA fragments. The in vitro reconstituted mono- and di-nucleosomes could be visualized. Thin arrows point to the reconstituted mono-nucleosomes and thick arrows indicate the di-nucleosomes.

$\triangleleft$ Fig 2. AFM images of the dynamic process of the in vitro nucleosome reconstitution. Samples of the in vitro nucleosomes reconstitution at different concentrations of $\mathrm{NaCl}$ were prepared and imaged with AFM. (A) The histones octamers were dissociated from DNA at $1 M \mathrm{NaCl}$. (B) And (C) the core histones bound to DNA gradually when the concentration was slowly reduced to $650 \mathrm{mM}$ and $300 \mathrm{mM}$. (D) The fibers were folded into the compact fibers at $100 \mathrm{~m} M \mathrm{NaCl}$. (E) The beads-on-a-string structure could be visualized at $50 \mathrm{~m} M \mathrm{NaCl}$. (F) The linker histones-stripped oligo-nucleosomes as a control.

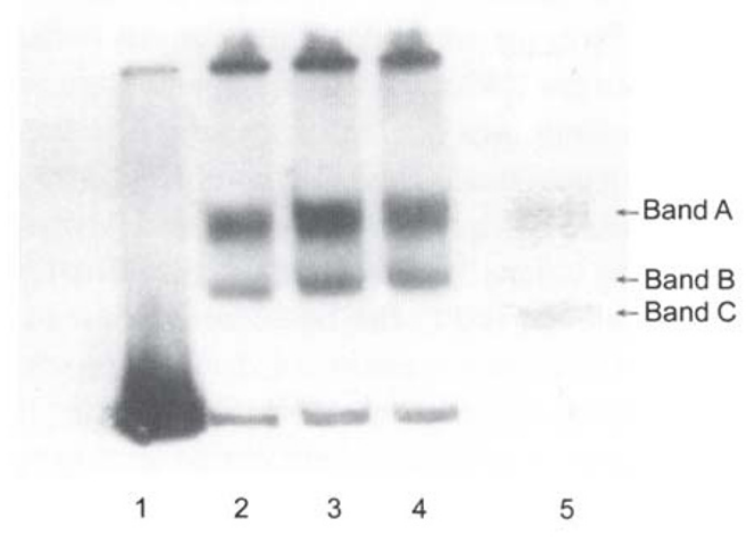

Fig 4. Identification of in vitro reconstitution of nucleosomes with the labeled HS2core DNA fragments. Lane 1, the naked labeled HScore DNA probe; lanes $2-4$, the in vitro reconstitution of nucleosomes: the ratios of the linker histones-stripped oligo-nucleosomes to labeled HS2core DNA are 100:1, 150:1, and 200:1 respectively; lane 5, the native mono-, di- nucleosomes prepared from chicken erythrocytes were stained with $\mathrm{EB}$ as markers.

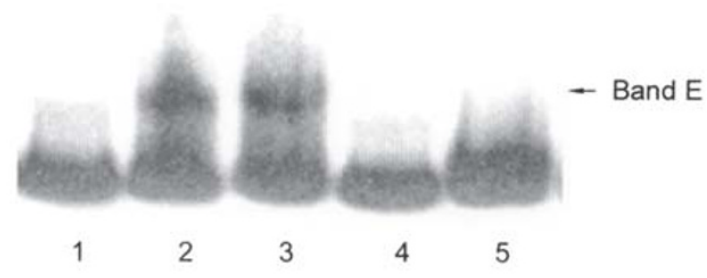

Fig 5. Gel mobility shift assay of HMG proteins binding to the naked HS2 core DNA sequence. Lane 1, labeled HS2core DNA fragment without HMG proteins; lanes 2 and 3, labeled HS2 core DNA fragment with 1.5 and $2 \mathrm{mg}$ of HMG (1/2) proteins respectively; lanes 4 and 5, labeled HS2 core DNA fragment with 1.5 and 2 mg of HMG $(14 / 17)$ proteins respectively.

The binding of HMG proteins to HS2core DNA sequence in $L C R$

In order to investigate the binding of $\mathrm{HMG}$ proteins (HMG1/2 and HMG14/17) to the HS2core DNA sequence presented in different states (either in the naked DNA or in the in vitro reconstituted nucleosomal DNA), Gel mobility shift assays were performed. When the naked HS2core DNA fragment was used as a probe we demonstrated that no band could be detected by addition of HMG 14/17 proteins (Fig 5, lanes 4-5). While, One shift band (band E) could be revealed by addition of HMG1/2 proteins (Fig 5, lanes 2-3, band E). Data showed that HMG1/2 proteins could bind to the naked HS2core 
DNA sequence, whereas the HMG14/17 proteins couldn't. Furthermore, both bands 'A' and 'B' (mono- and di -nucleosomes repesented respectively) could be visible when the HS2core DNA fragments were reconstituted in vitro into nucleosomes (Fig 6, Lanes 1). Moreover, a new band (band D) was observed by addition of HMG14/17 proteins and the band A was also retarded (Fig 6, lane 3), indicating that HMG14/17 proteins are able to bind to the HS2core DNA sequence reconstituted in vitro into nucleosomes. While, no change could be detected by addition of $\mathrm{HMG1} / 2$ proteins, indicating that these proteins are unable to bind to the in vitro reconstituted nucleosomes (Fig 6, lanes 4-5). It seems that dynamic control of human $\beta$-like globin gene expression requires the regulated assembly and disassembly of the architectural component- enhancer DNA complex.

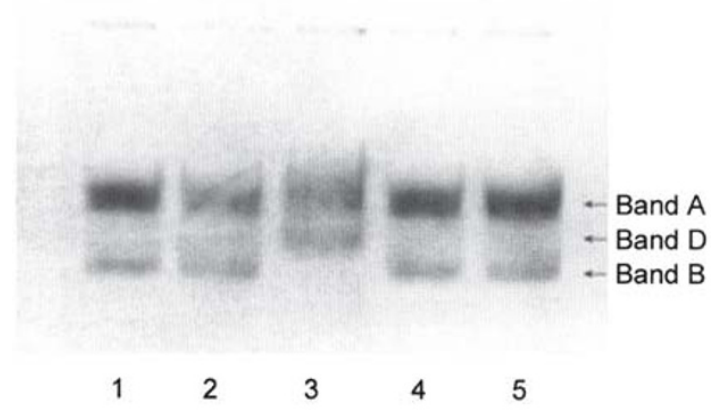

Fig 6. Gel mobility shift assay of HMG proteins binding to the nucleosomes reconstituted in vitro with labeled HS2core DNA fragment. Lane 1, the nucleosomes reconstituted in vitro with labeled HS2core DNA fragment; lanes 2 and 3, the nucleosomes reconstituted in vitro with labeled HS2core DNA fragment. 5 and $10 \mu \mathrm{g}$ of HMG14/17 proteins were added respectively; lanes 4 and 5, the nucleosomes reconstituted in vitro with labeled HS2 core DNA fragment. 5 and $10 \mu \mathrm{g}$ of HMG1/2 proteins were added respectively.

The binding of HMG proteins to NCR2 DNA sequence in 5' flanking DNA sequence of human b-globin gene

Next, we were interested in testing the binding of HMG proteins (HMG1/2 and HMG 14/17) to the NCR2 (a silencer, -372 to $-194 \mathrm{bp}, \sim 178 \mathrm{bp}$ ) in the 5 'flanking sequence of human b-globin gene[3]. The mononucleosomes were also reconstituted in vitro with this DNA fragment (Fig 7, lanes 1-2, band F). In addition, data further showed that HMG $1 / 2$ proteins could bind to the naked NCR2 DNA sequence (Fig 8, lane 1, band G),

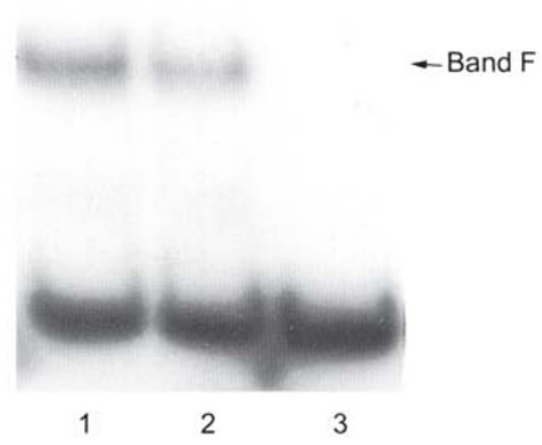

Fig 7. Identification of in vitro reconstitution of nucleosomes with labeled NCR2 DNA fragments. Lanes 1 and 2, in vitro reconstitution of mono-nucleosomes; the ratios of linker histones-stripped oligonucleosomes to labeled NCR2 DNA are 200:1 and 100:1 respectively; lane 3, naked labeled NCR2 DNA probe.
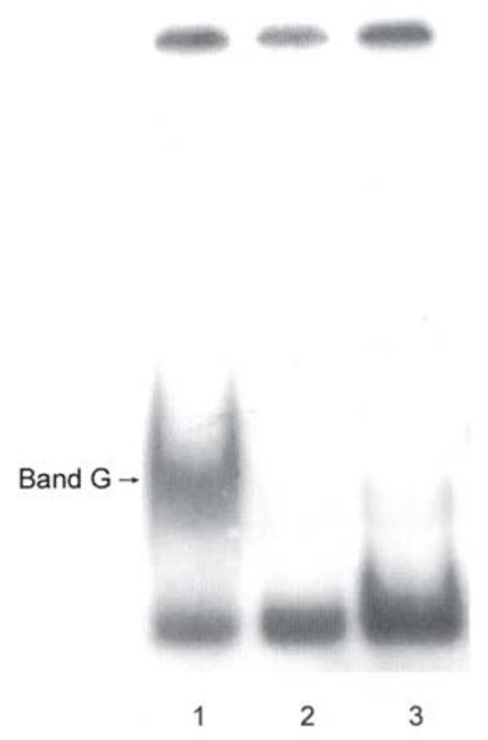

Fig 8. Gel mobility shift assay of HMG proteins (HMG1/2 and HMG14/17) to naked NCR2 DNA sequence (-372 to $-194 \mathrm{bp})$ in the 5 'flanking sequence of human b-globin gene. Lane 1, labeled NCR2 DNA fragment with $1 \mu \mathrm{g}$ of HMG1/2 proteins; lane 2, labeled NCR2 DNA fragment without HMG proteins; lane 3, labeled NCR2 DNA fragment with $1 \mu \mathrm{g}$ of HMG $14 / 17$ proteins

whereas HMG14/17 proteins couldn't (Fig 8, lane 3). In contrast, HMG14/17 proteins were capable of binding to the mono-nucleosome reconstituted in vitro with NCR2 
DNA fragment (Fig 9, lane 1, band H), whereas HMG1/ 2 proteins were unable to bind to it (Fig 9, lane 2). These results further confirmed that the binding of HMG proteins (HMG1/2 and HMG14/17) to the tested distal and proximal regulatory elements in the 5 'flanking sequence of human b-globin gene is dynamic following the assembly and disassembly of nucleosome. We suggest that the dynamic binding patterns of HMG proteins to the regulatory elements might be related to the regulation of the human b-globin gene expression.

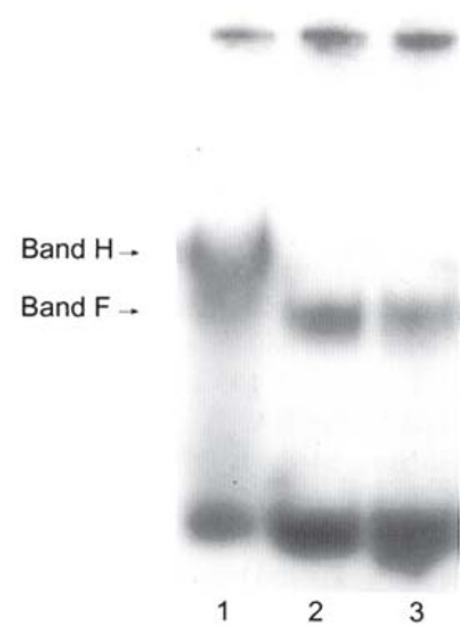

Fig 9. Gel mobility shift assay of HMG proteins (HMG1/2 and HMG14/17) binding to the mono-nucleosomes reconstituted in vitro with labeled NCR2 DNA fragment. Lane 1, mono-nucleosome reconstituted in vitro with labeled NCR2 DNA fragment, $5 \mu \mathrm{g}$ of HMG14/17 proteins was added; lane 2, mono-nucleosome reconstituted in vitro with labeled NCR2 DNA fragment, $5 \mu \mathrm{g}$ of HMG1/2 proteins was added; lane 3 , the mono-nucleosome reconstituted in vitro with labeled NCR2 DNA fragment.

\section{DISCUSSION}

The orderly progression of most DNA-related activities such as transcription, replication, recombination and repair involves changes in the structure of DNA and in the organization of chromatin fiber. To understand how a long extended DNA folds into a chromosome is one of the most important problems in biology. All eukaryotic chromosomes consist of a regularly repeating proteinDNA complex called the nucleosome[10]. It is traditionally considered to be the fundamental unit of chromatin structure. The X-ray crystal structure of the complete nucleosome core particle was resolved at 2.8 ? resolution, providing a detailed view of the protein and DNA organization[11].

Recently, the atomic force microscopy (AFM) has been used for exploring the structure of chromatin[12, 13]. The technique of AFM has several advantages over more classical imaging techniques like EM. It allows imaging of biological macromolecules and structures in ambient conditions, without staining or shadowing, in air or under liquids. Therefore, AFM can image biological macromolecules under conditions close to their native condition. It provides more clearly images of the structure of chromatin than that attained by electron microscopy. Using the atomic force microscopy, we have previously revealed the structure of the nucleosome core particle of chromatin in chicken erythrocytes: the $\sim 146 \mathrm{bp}$ of DNA wrapped twice around the core histone octamer were clearly visualized. Both the ends of entry /exit of linker DNA were also demonstrated. The dimension of the nucleosome core particles is $\sim 1-4 \mathrm{~nm}$ in height and $\sim 13-22 \mathrm{~nm}$ in width[2].

Nucleosomes and chromatin fibers are dynamic, not inert. Their dynamic behavior is essential for chromatin function. Most DNA in vivo is packaged in nucleosomes, and many DNA sequences are critical for biological regulation and so must be accessible to regulatory proteins at appropriate times. What are the principles that guarantee that regulatory proteins will have access to their DNA target sequences when necessary?

In an attempt to gain further insight into the dynamic processes of in vitro nucleosome reconstitution, the linker histones-depleted oligo-nucleosomes were prepared from chicken erythrocytes. The dynamic process of in vitro nucleosome reconstitution followed by slow dilution from high salt to low salt was visualized by AFM. Once the salt concentration has been reduced to $50 \mathrm{mM}$, an extended beads-on-a-string structure could be clearly visualized (height of $\sim 2-4 \mathrm{~nm}$, width of $\sim 18 \mathrm{~nm}$ for each individual nucleosome).

Gene-Specifc transcriptional switches are thought to be generated through the dynamic assembly and disassembly of transcription factor-enhancer DNA complex. The high mobility group proteins (HMG proteins) are a set of non-histone proteins in the nuclei of all higher eukaryotes. However, the biological functions of these proteins are poorly understood. They are generally considered to function as architectural elements that modify the structure of DNA and chromatin to generate a conformation for regulating various DNA-dependant activities. HMG 14/17 proteins are the only nuclear proteins known to specifically recognize the generic structure of the 146 bp-nucleosome core[14-16]. They are 
modestly enriched in the actively transcribed gene relative to the inactive gene[16-19]. It was known that HMG 14/17 proteins could enhance the replication and transcription when the chromatin was used as template[20].

We previously revealed that HMG proteins (HMG1/ 2 and HMG14/17) could specifically interact with the regulatory elements in the 5 'flanking sequence of the human e-globin gene and suggested that these proteins might participate in the regulation of this gene's expression[21]. In addition, Our previous studies demonstrated that HMG1/2 proteins were able to bind to a negative regulatory region NCR1 (-618 to $-373 \mathrm{bp}$ ) in the 5'flanking sequence of human b-globin gene. Using scanning tunneling microscopy (STM) we revealed that the binding of HMG1/2 to NCR1 could bend this DNA fragment to form a circular structure with a linear tail[22]. Therefore, we tend to conclude that the changes of the local DNA conformation induced by HMG1/2 proteins may play an important role in regulating the expression of human b-globin gene.

In order to further investigate the interactions between $\mathrm{HMG}$ proteins (HMG1/2 and HMG14/17 proteins) and the HS2core DNA sequence in LCR, the in vitro reconstitution of nucleosomes with the $\gamma-32 \mathrm{P}-$ labeled and unlabeled HS2core DNA fragments was carried out. Results showed in EMSA that HMG 1/2 proteins could bind to the naked HS2core DNA sequence. While, HMG14/17 proteins could not. Furthermore, HMG14/17 proteins were capable of binding to the mono- and di nucleosomes reconstituted in vitro with HS2core DNA sequence. In contrast, HMG1/2 were unable to bind to them. Meantime, the NCR2 (-372 to -194 bp) in the 5 'flanking sequence of human b-globin gene was also assembled in vitro into mono-nucleosomes. Data showed that the binding patterns of HMG1/2 and HMG14/17 proteins to the NCR2 presented in different states (either in the naked DNA or in the in vitro reconstituted nucleosomal DNA) were consistent with that of HS2core sequences. We suggest that the changeable binding patterns of HMG1/2 and HMG14/17 proteins with the regulatory elements may be critical in the process of nucleosome assembly, recruitment of chromatin-modifying activities, and the regulation of human $\beta$-globin gene expression.

\section{AKNOWLEDGEMENT}

This work was supported by the National Natural
Science Foundation of China (Grant No. 39893320 and 39870378) and the Foundation of the Chinese Academy of Sciences (Grant No. Kj982-j1-618).

\section{REFERENCES}

1 Qian RL, Liu ZX, Zhou MY, Xie HY, Jiang C, Yan ZJ, Li MQ, Zhang Y, and $\mathrm{Hu}$ J. Visualization of chromatin folding patterns in chicken erythrocytes by atomic force microscopy (AFM). Cell Research 1997; 7:143-50.

2 Zhao H, Zhang Y, Zhang SB, Jiang C, He QY, Li MQ, and Qian $\mathrm{RL}$. The structure of the nucleosome core particle of chromatin in chicken erythrocytes visualized by using atomic force microscopy. Cell Research 1999; 9:255-60.

3 Berg, PE, Williams DM, Qian RL, Cohen Rb, Cao SX, Mittelman $\mathrm{M}$, and Schechter AN. A common protein binds to two silencers 5 'to the human b-globin gene. Nucleic Acids Res 1989; 17:883352.

4 Lutter LC. Kinetic analysis of deoxyribonuclease I cleavages in the nucleosome core: evidence for a DNA superhelix. J Mol Biol 1978; 124:391-420.

5 Drew HR and Calladine CR. Sequence-specific positioning of core histones on an 860 base-pair DNA, experiment and theory. J Mol Biol 1987; 195:143-73.

6 Rhodes D and Laskey RA. Assembly of nucleosomes and chromatin in vitro. Methods Enzymol 1989; 170:575-85.

7 Goodwin GH and Johns EW.Isolation and characterization of two calf-thymus chromotin non-histone proteins with high contents of acidic and basic amino acids. Eur J Biochem 1973; 40:215-9.

8 Bradford MM. A rapid and sensitive method for the quantitation of microgram quantities of protein utilizing the principle of protein-dye binding. Anal Biochem 1976; 72:248-54.

9 Yan ZJ, Chen YD, and Qian RL. Developmental stage-specific factors in the mouse hematopoietic tissues binding to the 5'flanking cis-acting element of human e-globin gene. Chinese Science Bulletin 1995; 40:778-83.

10 Kornberg RD. Structure of chromatin. Annu Rev Biochem 1977; 46:931-54.

11 Luger K, Mader AW, Richmond RK, Sargent DF, and Richmond TJ. Crystal structure of the nucleosome core particle at 2.8 ? resolution. Nature 1997; 389:251-60.

12 Allen MJ, Dong XF, O'Neill TE, Yau P, Kowalczykowski SC, Gatewood J, Balhorn R and Bradbury EM. Atomic force microscope measurement of nucleosome cores assembled along defined DNA sequences. Biochemistry 1993; 32:8390-6.

13 Leuba SH, Bustamante C, Van holde K and Zlatanova J. Linker histone tails and $\mathrm{N}$-tails of histone $\mathrm{H} 3$ are redundant: Scanning force microscopy studies of reconstituted fibers. Biophys J 1998; 74:2830-9.

14 Bustin M. and Reeves R. High mobility group chromosomal proteins: architectural components that facilitate chromatin function. Prog Nucleic Acid Res Mol Biol 1996; 54:35-100.

15 Michael B and Raymond R. High-mobility-group chromosomal proteins: architectural components that facilitate chromatin function. Progress in Nucleic Acid Research and Molecular Biology 1996; 54:35-101. 
16 Sandeen G, Wood WI and Felsenfeld G. The interaction of high mobility proteins HMG14 and 17 with nucleosomes. Nucleic Acids Res 1980; 8:3757-78.

17 Druckmann S, Mendelson E, Landsman D and Bustin M. Immunofractionation of DNA sequences associated with HMG 17 in chromatin. Exp Cell Research 1986; 166:486-96.

18 Brotherton TW, Reneker J and Ginder GD. Binding of HMG 17 to mononucleosomes of the avian b-globin gene cluster in erythroid and non-erythroid cells. Nucleic Acids Res 1990; 18:2011-6.

19 Postnikov YV, Shick VV, Belyavsky AV, Khrapko KR, Brodolin KL, Nikolskaya TA and Mirzabekov AD. Distribution of high mobility group proteins $1 / 2, \mathrm{E}$ and $14 / 17$ and linker histones H1 and $\mathrm{H} 5$ on transcribed and non-transcribed regions of chicken erythrocyte chromatin. Nucleic Acids Res 1991; 19:717-25.

20 Trieschmann L, Alfonso PJ, Crippa MP, Wolffe AP and Bustin M. Incorporation of chromosomal proteins HMG-14/HMG-17 into nascent nucleosomes induces an extended chromatin conformation and enhances the utilization of active transcription complexes. EMBO J 1995; 14:1478-89.

21 Yan ZJ, Chen YD and Qian RL. Interactions between HMG proteins and 5'flanking cis-acting elements of human e-globin gene. Acta Biochimica et Biophysica Sinica 1998; 30:471-5.

22 Qian RL, Chen YD, Hu YL, Li MQ, Wang ZH and Xu YL. Interaction between $\mathrm{HMG}$ proteins $(1+2)$ and the negative regulatory region 1 (NCR1) in the 5'-flanking sequence of the human b-globin gene. Science in China (series B) 1993; 36:933-9. 